\title{
Workshop II. Diagnosis of Reiter's syndrome among B27-associated and other seronegative spondarthropathies
}

\section{Introduction}

\author{
F. C. ARNETT
}

From the Johns Hopkins University and the Good Samaritan Hospital, Maryland, USA

As we face the diagnostic difficulties among seronegative B27-associated arthropathies-the topic of Workshop II-we are confronted with the philosophical and practical problem of 'lumping' or 'splitting' clinical syndromes. By personal bias I am an affirmed 'splitter'. Indeed, unless homogeneous clinical populations are carefully defined prior to any aetiological study important clues might be discarded on the basis of negative results in a proportion of patients. The value of clinical 'splitting' has become particularly obvious in diseases such as the mucopolysaccharidoses where precise clinical separation was required before elucidation of the biochemical defects.

Even for the mere diagnosis typical questions will arise. Is incomplete Reiter's syndrome (RS) the same as the classical triad? Where does RS end and ankylosing spondylitis (AS) begin (as already discussed in Workshop I)? Are RS and AS two facets of a single entity at different stages of disease activity (Fig. 1)? Has RS got the 'choice' to burn out without any sacroiliitis, or, as an alternative, to recur with eventual appearance of sacroiliitis or

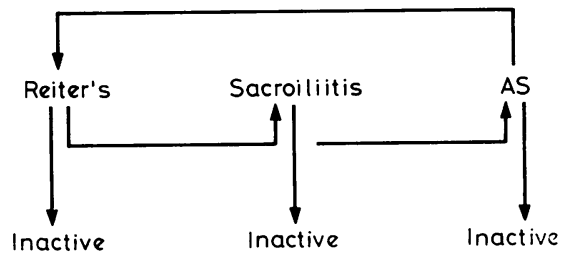

Fig. 1 Hypothetical relationships between Reiter's disease and ankylosing spondylitis. (Spectrum of disease or several diseases.) full-blown AS (as Dr. Woodrow had elegantly described it)? Professor Wright, who is a firm 'splitter', will probably comment on the relationship of keratodermia to psoriasis, if any. Can psoriasis or its genes turn on RS the way it appears, in some HLA-B27-negative patients, to turn on AS?

I would like to present three illustrative cases of psoriasis and arthritis.

Firstly, a 30-year-old man who at the age of 26 developed a mild dysenteric illness followed by severe polyarthritis, keratodermia, oral ulcers, balanitis, fever, and a weight loss of $30 \mathrm{lb}(13.6 \mathrm{~kg})$ but who does not have any urethritis or conjunctivitis. Two years later, when aged 28 , his arthritis remitted. His skin disease, however, continued with the appearance of an extensive psoriasiform rash and persisted with exfoliation. Now, at the age of 30 , he is still exfoliating and his rash is controlled only with methotrexate therapy. At this stage he has an episode of iritis, typical nail changes, balanitis, and recurring arthritis-in short, the problem of typical keratodermia blennorrhagica progressing towards a generalised psoriatical rash requiring methotrexate therapy eventually. Genetically (Fig. 2) he is B27-positive without any of the HLA antigens (W17, B13, B38) more typically associated with psoriatic arthropathy. His pedigree is unfortunately uninformative. One B locus of his diseased relatives could not be identified at the time. Did this patient have psoriatic arthritis at the onset or is it a case of RS developing into full-blown AS?

The second patient is one who was admitted to the Johns Hopkins Hospital at the age of 2 with severe conjunctivitis associated with arthritis in his knees and ankles. As a result of heel pain he walked on his 


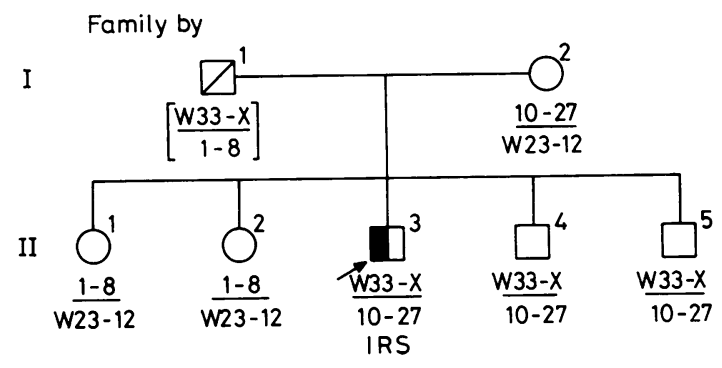

Fig. 2 Genotype and pedigree of patient with both Reiter's disease and psoriatic arthropathy.

toes and had tenderness on palpation of his heels. By the age of 15 he developed typical psoriasis. When aged 32 he began to suffer from episodic arthritis of the knees, ankles, toes, wrists, and hip with heel pain and fever. Finally, when we saw him at the age of 47 he had severe arthritis and psoriasis, typical pitting of the nails, yet no DIP involvement. Radiographs showed typical periostitis of the calcanei and sacroiliitis. He had received the B27 from one parent and the BW38 from the other. His parents are healthy.

The third patient is a medical student who, when aged 23 , started a mild psoriaform rash over the scalp. Later he developed arthritis suggestive of RS with asymmetric involvement of the toes. The first toe to be affected was removed by an orthopaedic surgeon because he thought it was a tumour. After several toes had been affected heel pain, balanitis, painless oral ulcers, and weight loss developed. Only then did he develop urethritis. He now suffers from heel pain but has no sacroiliitis, and despite absence of nail pitting or DIP involvement he is a carrier of the antigen BW38 but not B27. Is this psoriatic arthritis or RS, or, has a gene of psoriasis turned on a Reiter's-like illness without need of B27? Should the initial disease be classified as juvenile chronic polyarthritis?

Indeed, we have seen a B27-positive woman who started the symmetrical rheumatoid-like polyarthritis with low back pain at the age of 15 . When we saw her at the age of 59 she had destructive erosive seropositive arthritis in both hands and fusion of the entire spine.

As one can see, the clinical difficulties may be considerable. This present workshop will discuss some of the discriminating features to try to distinguish RS, AS with peripheral arthritis, psoriatic arthropathy, gonococcal arthritis, B27positive juvenile chronic arthritis, and-if such entity exists-the so-called 'seronegative' rheumatoid arthritis.

\section{General Discussion}

PROF. M. ZIFF: In everybody's experience here there are patients (about 24 reported cases) who show aspects of psoriatic arthropathy and RS often sequentially. You cannot really split or categorise them as they must have something in common.

DR. ARNETT: Yes, let's keep an open mind about them. Put the pure Reiter's together and study them genetically; do the same for the pure psoriatics. Study these overlap patients in a search for multigenetic factors as exemplified by the second patient I presented.

PROF. A. E. GOOD: I persist in my instinct to go on splitting. We see such disparate diseases as rheumatoid arthritis and classical ankylosing spondylitis in the same patient more often as we look for them. I am not perturbed by the coexistence of psoriasis with features of another disease too.

DR. ARNETT: Of course, $2 \%$ of the population has psoriasis. In every 100 patients with RS one would expect two to have psoriasis, just as was pointed out for rheumatoid arthritis.

DR. E. ALBERT: I also am a 'splitter', and with the discovery of HLA antigens splitting has proved quite successful. We have split diseases and we have split antigenic specificities. I would like to quote Roy Watford, one of the enfants terribles authorities in the field of HLA, who said: 'We are going on to split until the antigens eventually disappear.' Splitting diseases, however, will not make them disappear but yield better definitions. On the other hand, we might have to be very careful about splitting too far. For B27-associated diseases, for example, we have to take into account a very large variation of expression within any one of the associated diseases. Variability versus multiplicity of diseases will eventually be settled by how much variability we are going to see expressed within a population and within a family. We ought to test the variability of expressions of a given disease in monozygotic twins as compared to the same diseases in dizygotic twins or families. 